Revue internationale de pédagogie de

l'enseignement supérieur

$36(2) \mid 2020$

Numéro spécial - été 2020

\title{
Le carnet réflexif partagé : un espace d'écriture heuristique
}

Marlène Lebrun

\section{(2) OpenEdition}

1 Journals

Electronic version

URL: http://journals.openedition.org/ripes/2677

DOI: 10.4000/ripes.2677

ISSN: 2076-8427

Publisher

Association internationale de pédagogie universitaire

Electronic reference

Marlène Lebrun, "Le carnet réflexif partagé : un espace d'écriture heuristique ", Revue internationale de pédagogie de l'enseignement supérieur [Online], 36(2) | 2020, Online since 22 November 2020,

connection on 29 January 2021. URL: http://journals.openedition.org/ripes/2677 ; DOI: https://doi.org/ $10.4000 /$ ripes. 2677

This text was automatically generated on 29 January 2021.

Article L.111-1 du Code de la propriété intellectuelle. 


\title{
Le carnet réflexif partagé : un espace d'écriture heuristique
}

\author{
Marlène Lebrun
}

\section{Introduction}

1 Le propos consiste à analyser l'intérêt d'un outil de réflexivité pour l'enseignement tertiaire et la formation à l'enseignement en présentant ses tenants et aboutissants. Après une présentation du cadrage théorique et des modalités d'implémentation de l'outil d'écriture, les représentations des étudiants qui ont mis en œuvre cet outil dans leur formation seront analysées pour mettre en évidence que le va-et-vient entre empathie et distanciation est une condition de la réflexivité dans et par l'écriture.

\section{Cadrage théorique : pourquoi le CRP?}

2 Décrire la genèse du CRP permet de comprendre les conceptions en amont de l'expérimentation d'un outil d'écriture partagée que j'ai modélisé dès 2010 et que j'ai fait évoluer en fonction des publics d'enseignement et de formation.

Dans le cadre des recherches que j'ai menées en didactique de la littérature, j'ai souhaité constituer la classe de français comme une communauté de lecteurs et d'auteurs en dialogue, afin d'optimiser le développement des compétences liées en lecture et en écriture, autrement dit l'acculturation écrite (Lebrun, 2014). Quand je parle de compétences, j'englobe le terme d'appétences, non dans le sens d'un pré requis motivationnel mais dans celui d'une conséquence liée au développement des compétences en jeu. Autrement dit, le plaisir de lire et celui d'écrire ne sont pas des pré-donnés mais des résultantes, des sortes d'endomorphines cognitives.

Dans cette perspective, j'ai modélisé, entre autres, deux outils didactiques, à savoir le journal de lecture dialogué ou cahier culturel partagé et le cahier d'écrivain (Lebrun, 2010). Pour le premier, il s'agit de partager ses hypothèses de lecture et ses interprétations à propos des textes littéraires lus dans l'espace de la classe. L'écriture 
métatextuelle, c'est-à-dire une écriture critique sur les textes, est travaillée dans et par le partage. Le cahier culturel partagé concerne toutes les manifestations culturelles, pas seulement celles littéraires, mais aussi celles artistiques, théâtrales ou encore cinématographiques. Il donne vie à la culture vécue dans l'espace de la classe qui promeut les débats interprétatifs.

5 Enfin le cahier d'écrivain concerne le partage d'une écriture hypertextuelle (à partir des textes littéraires) ou créative de type fictionnel ou poétique. Le fait que le commentaire critique ou la production textuelle soit lue par les pairs, qui constituent un véritable lectorat avec une attention esthétique qui s'exprime dans un jugement de goût et de valeur, donne de la légitimité au texte qui n'a pas pour seule fonction d'être écrit pour être évalué par un enseignant à l'aune des normes génériques et linguistiques attendues. Aussi, l'espace de la classe se constitue-t-il comme un espace qui autorise et « auteurise ». Le scripteur s'autorise à écrire car il est vraiment lu et pas simplement évalué dans un cadre scolaire. Le néologisme «auteuriser» est un motvalise qui réunit auteur et autorisation.

6 En me fondant sur les thèses des sociologues de l'éducation (Lahire, 2004) et les didacticiens qui ont travaillé sur la dimension du sujet et le rapport à l'écrit (Barré de Miniac, 2002; Langlade et Rouxel, 2004), j’ai élaboré des dispositifs didactiques fondés sur le partage permettant de développer une posture critique, auctoriale et réflexive (Lebrun, 2010; 2014).

7 Il importe de définir ces trois adjectifs accolés au terme de posture auquel je donne la définition suivante. Une posture est un schème comportemental évolutif lié à la vie relationnelle et intellectuelle. Une posture implique un rapport à un objet et à un environnement; elle est propre au sujet qui la construit, plus ou moins à son insu.

Critique vient du grec "krinein" qui signifie formuler un jugement. Adopter une posture critique suppose donc d'être capable de juger en connaissance de cause, c'est-àdire avec pertinence. Lorsque nous assistons à un concert ou que visionnons un film, nous formulons un jugement de goût (cela me touche car....j'aime ou je n'aime pas parce que....) et de valeur (cela me fait penser à....) qui convoque notre bibliothèque personnelle, autrement dit notre réseau de références culturelles. Il en va de même pour la critique d'un texte qu'il soit littéraire ou journalistique. Souvent, les étudiants ont développé des compétences culturelles sur les œuvres contemporaines qui les interpellent mais elles et ils ne pensent pas ou ne s'autorisent pas à les transférer sur d'autres domaines culturels et d'autres œuvres, notamment littéraires et patrimoniales.

La posture auctoriale ou d'auteur est en lien avec la posture critique car pour critiquer au sens précisé "supra", il faut s'y autoriser, ce qui correspond à l'étymologie d'auteur, « auctor » en latin. Le lecteur qui lit autorise celui ou celle qui a écrit à écrire pour être véritablement lu. Ainsi, s'autorisera-t-il à écrire grâce à l'auctorialisation des pairs et de l'enseignant qui a une attention esthétique et critique pas phagocytée par celle des normes linguistiques qui empêchent d'écrire faute de maîtrise, pour peu que la maîtrise en matière linguistique existe réellement.

10 Enfin la réflexivité est un processus transformateur des représentations, des pratiques sociales, des savoirs. Une posture réflexive est relative au retour de la pensée sur ellemême. C'est bien l'image du miroir qui réfléchit. Si l'on revient aux origines du concept de réflexivité, on remonte à John Dewey (1968), philosophe et pédagogue américain, qui considère que l'intervention d'un enseignant est/devrait être le fruit d'un processus de réflexion qui puisse lui permettre de justifier, de planifier et de prévoir les 
conséquences de son action d'enseignement. Plus connu est le concept du praticien réflexif vulgarisé par Donald A. Schön (1993) dans la lignée des travaux fondateurs de Dewey. De son point de vue de praticien chercheur, Schön constate de fait une rupture entre les savoirs issus de l'expérience professionnelle et ceux issus des connaissances scientifiques. C'est la difficulté de faire un lien entre les deux types de savoirs (le fameux clivage théorie/pratique) et de prendre en compte les savoirs appropriés (beaucoup plus importants que le praticien ne le laisse paraitre). John Dewey parlait de l'importance de la continuité de l'expérience professionnelle pour pallier la rupture en question. J'ajoute que c'est d'autant plus important si l'on souhaite que les praticiens aient, non pas des croyances, mais des connaissances.

11 Ainsi l'analyse réflexive est-elle un processus cognitif continu qui s'apprend et s'exerce non seulement dans le cours de l'action (résolution de problèmes pédagogiques et didactiques en faisant preuve de créativité) mais aussi après l'action (capacité de distanciation et posture critique qui alimente la créativité). Les travaux de Schön sont à l'origine de la vogue des communautés professionnelles où les praticiens partagent et échangent leurs pratiques constituées en savoirs d'expériences (cercles de pratiques).

12 En sciences de l'éducation, de nombreux chercheurs ont travaillé à partir du concept de Schön. L'intégration d'une approche réflexive dans la pratique aide à prendre des décisions plus éclairées et suscite, chez l'enseignant, le souci de s'interroger sur son rôle dans la classe et dans la société. Wentzel (2014) prône l'intégration d'une approche réflexive dans la formation à l'enseignement pour que l'enseignant se constitue comme un professionnel- des apprentissages pour reprendre l'expression de P. Meirieu.

13 Serait-ce la finalité du CRP tel que le précise l'étudiant Anthony ${ }^{1}$ qui l'a expérimenté en cours de Sciences de l'éducation dans son cursus de Bachelor, d'abord à son corps défendant puisqu'il dit préférer le discours oral au discours écrit, puis avec beaucoup d'intérêt :

14 «J'ai beaucoup aimé l'idée du cahier d'écriture réflexif partagé. D’une part, il m'a permis de pouvoir me situer idéologiquement et politiquement face à mes futurs collègues -ce qui est à mon sens important pour travailler en collaboration avec le corps enseignant que je rejoindrai bientôt- et d'autre part il m'a donné la possibilité d'approfondir mes propres réflexions parfois trop globales sur certaines thématiques. Le fait d'écrire pousse automatiquement à réarranger ses idées pour qu'elles prennent forme et deviennent cohérentes. Étant plus volontiers une personne oralisante, cette méthode de travail m'a aidé à ordonner mes pensées parfois trop chaotiques. »

L'étudiant ajoute que le cours accompagné de l'outil lui a permis de/d' :

16 - connaître l'avis de mes pairs sur les grandes questions que pose et que se pose l'école aujourd'hui;

17 - approfondir mon bagage de connaissances pour avoir un œil éclairé sur les enjeux de l'actualité scolaire;

18 - pouvoir prendre position en argumentant avec des références à l'appui.

19 En tant qu'initiatrice de cet outil d'enseignement dans un cours de Sciences de l'éducation au niveau bachelor', les commentaires d'Anthony me confortent dans les enjeux que j'avais assignés au CRP, à savoir, s'autoriser à penser.

Le cours est accompagné par un outil d'écriture, le CRP qui aide les étudiants à exprimer par écrit leurs représentations, conceptions, idées, réactions et commentaires à partir de questions vives en lien avec le plan de cours. Les offres d'écriture sont 
informelles et accompagnent le cours de manière diverse et variée. Le partage des textes et des commentaires du CRP promeuvent une interactivité proche de la pédagogie inversée.

21 Une posture réflexive permet de faire des liens entre l'action quotidienne et la compréhension de son action pour être capable de décrire, d'analyser, de critiquer et d'innover à l'intérieur de sa démarche d'enseignement. Cette posture suppose aussi une posture critique et une posture auctoriale, telles que définies supra, qui s'alimentent mutuellement. Il est loisible de comprendre qu'une posture critique, auctoriale et réflexive conjointe favorise l'autonomisation des étudiants dans l'écriture en leur permettant d'associer vouloir et pouvoir, appétence et compétence.

Peut-être est-il utile, à ce stade de la réflexion, de décrire plus précisément les modalités de mise en œuvre de l'outil pour le lecteur intéressé.

\section{Modalités de mise en œuvre : le CRP comment?}

Le carnet réflexif est un outil d'écriture heuristique qui permet de construire sa pensée tout en déconstruisant ses représentations liminaires. Le professeur propose une question ouverte (voir annexes 2 et 3) et l'étudiant y répond d'emblée en fonction de ses représentations et savoirs déjà-là. Le carnet réflexif est lu par les pairs qui entrent dans le dialogue de la pensée, dans le débat et commentent et/ ou questionnent l'auteur. Si la page de gauche est réservée à l'auteur du journal, la page de vis-à-vis, soit de droite est réservée aux pairs. L'utilisation de l'outil est très souple et dépend de la dynamique du cours. L'intérêt est d'aider ou d'accompagner l'étudiant à construire une réflexion. L'écriture est heuristique au sens où elle est une écriture de travail et de tâtonnement qui n'est pas destinée à être évaluée par l'enseignant. Cet outil d'écriture partagée permet aussi de prendre conscience de l'évolution de ses savoirs et de sa réflexion puisque la question est reprise à différents moments. L'étudiant note toujours la date de son écriture qu'elle soit commentative ou non, cela favorise sa relecture et l'appréhension de la genèse de son écriture et de l'évolution de sa pensée.

24 A l'introduction de l'outil, il importe d'insister sur la fonction heuristique de l'écriture. Pour débloquer et motiver les étudiants résistants (et assez nombreux à l'être) au début du cours, j'ai précisé que les écrits prenaient la forme de traces, c'est-à-dire de premiers jets dont la forme n'est pas achevée. Le seul critère de réussite est celui de la lisibilité pour favoriser le dialogue et le débat avec les pairs. La résistance ou le blocage vient d'une représentation de l'écriture comme la traduction d'un pré-donné qui suppose don et inspiration. En outre, l'évaluation scolaire de l'écriture, le plus souvent sommative et certificative, minore les écrits de travail ou intermédiaires.

La spécificité du CRP réside dans le partage avec les pairs qui commentent, questionnent et entrent dans le débat. C'est ce dialogue pluriel qui force et autorise à penser avec l'écrit et par l'écrit. Il s'agit d'une fonction heuristique de l'écrit qui est une verbalisation favorisant la distanciation et la découverte de sa propre pensée qui ne préexiste pas au langage en tant que « raison graphique » (Goody, 1979).

L'outil d'écriture accompagne l'étudiant de Bachelor tout au long d'un cours de sciences de l'éducation qui fait partie du cursus de formation professionnalisante à l'enseignement primaire dans une Haute École Pédagogique (HEP) en Suisse romande. Cette formation dure trois années. Constitué de 24 périodes sur un semestre, le cours, 
dont sont tirés la plupart des verbatims analysés dans cette contribution, s'intitule "Promouvoir un enseignement qui favorise la réussite de tous les élèves ». Il vaut 4 crédits ECTS et l'évaluation se fait à travers un examen écrit de 2 heures proposant à l'étudiant 4 questions aux choix dont il en retient deux pour construire une réflexion structurée illustrée de relations empruntées à l'expérience professionnelle. Les questions sont ouvertes et vives, c'est-à-dire qu'elles appellent le débat.

Peut-être est-il utile de préciser que le temps de cours imparti à l'outil varie à chaque séance, qu'il n'est pas obligatoirement planifié, ni les questions d'ailleurs. Sur un cours de 2 périodes (soit $90 \mathrm{~min}$ ), voire de 4 (ce que j'apprécie particulièrement car l'apprentissage demande du temps et ne rime pas avec "zapping »), le temps d'écriture dans le CRP varie de 5 à 30 min et n'exclut pas d'autres formes d'écriture individuelle ou collective plus structurées qui font l'objet d'un apprentissage explicite.

\section{La parole au corps estudiantin en formation à l'enseignement : leur vécu à propos de l'outil d'écriture partagée}

\subsection{Quelques aspects méthodologiques}

Les données dont l'analyse fait l'objet de cette contribution ont été recueillies dans le cadre de cours de sciences de l'éducation dans un cursus de formation à l'enseignement primaire (bachelor) et secondaire (master) pendant deux années consécutives de 2016 à 2018. À la fin du cours, les étudiants volontaires (une soixantaine) ont été invités à remplir un questionnaire (voir annexe 1) ouvert sur l'outil d'écriture qui a accompagné le cours. Il est à noter que des moments de régulation en cours ont déjà été proposés sous forme de courts débats afin que l'outil soit l'objet d'échanges sur son intérêt et ses enjeux.

Les stratégies et les instruments de recueil des données issus d'une méthodologie mixte, ainsi que l'analyse qui en découle, permettent de rendre compte de la complexité et de la richesse des données récoltées (Poisson, 1983).

Par le recours à une recherche de type qualitatif, le chercheur aspire à la compréhension interne d'une situation dans laquelle il cherche à agir sur l'environnement en vue de transformation d'un réel; la recherche de type quantitatif est une façon de mesurer de façon externe ce qui se fait dans une zone plus large que celle du groupe expérimental de la recherche action. Il est vrai que les deux approches n'ont pas la même logique ni les mêmes outils mais un jumelage peut être bénéfique et fructueux d'un point de vue didactique car il peut renseigner sur la réalité, les connaissances que l'on peut construire à partir d'une situation donnée et ouvrir la voie à de nouvelles pistes d'exploitation pédagogique.

Blanchet (2014) pense que le quantitatif relève d'un travail qualitatif qui repose sur l'interprétation des données recueillies. «Au fond, le quantitatif est du qualitatif. D'une part, tout dépend de la façon dont on définit, dont on identifie et dont on va chercher ce que l'on compte: les questions et les catégories selon lesquelles les "données » quantitatives sont " recueillies » sont des éléments signifiants, issus d'interprétations préalables. D'autre part, de toute façon, les chiffres ne disent rien en eux-mêmes : il reste à les interpréter, c'est-à-dire à leur donner du sens, à les contextualiser, même 
dans une démarche statistique qui commence par « interroger les données » selon des procédures mathématiques et non selon des procédures qualitatives » (Blanchet, 2014, p. 12)

Le recours au questionnaire et son traitement par un outil informatique $\mathrm{SPSS}^{3}$ enrichit la recherche de type qualitatif et favorise une analyse fine des données, ce qui conduit à avoir une vision sur le rapport au CRP tel qu'il est perçu par les étudiants qui ont vécu la pratique d'écriture pendant un cours. La posture d'un praticien chercheur qui entre en interaction avec les participants du milieu d'étude, soit un cours de Bachelor, consiste à tisser des liens, à les observer dans une durée limitée, à les discuter afin de comprendre la dynamique à l'étude et évaluer l'évolution du rapport à l'écriture et la construction des compétences réflexives en jeu. Le questionnaire écrit est une méthode collective de recueil des données traitées par une analyse de contenu (Bardin, 1998) qui est un instrument méthodologique servant à étayer une argumentation et tirer des conclusions. L'analyse de contenu est fondée sur un travail d'interprétation qui tend vers l'objectivité scientifique par une description objective, systématique et quantitative des corpus objet de l'étude: «S'inscrire dans une démarche d'analyse de contenu donne une valeur scientifique au travail qualitatif en essayant de s'écarter le plus possible des "dangers de la compréhension spontanée" »(Bardin, 1998, p. 31).

Trois étapes caractérisent ce type d'analyse : la préanalyse, l'exploitation du matériel et le traitement des résultats par inférence et interprétation.

\subsection{Quelques résultats}

Nombre d'étudiants ${ }^{4}$ ont perçu la fonction heuristique de l'outil :

«Très intéressant! Au départ, je me suis dit que le carnet était sclérosé et unifiait les pensées. Or, au contraire, c'est un outil évaluatif et progressif! Le plus intéressant n'est peut-être pas sa réflexion! Mais la compréhension des autres réflexions, et d'apporter un regard nouveau sur la thématique. Cet outil collaboratif est un moyen d'échange et de progression des apprentissages. "

"C'est chouette de pouvoir exprimer son opinion et d'avoir des retours de la part des pairs. C'est bien d'avoir du temps en classe pour écrire. »

"Cela m'a appris à échanger, à débattre, à s'opposer dans un contexte en lien avec une réflexion professionnelle. Intéressant de rediriger sa réflexion grâce aux autres. »

"J'ai aimé découvrir ce que les autres avaient noté.... »

«J'ai adoré le concept. J'ai beaucoup appris à travers les réflexions de mes collègues. »

«J'ai appris que les autres nous apportent énormément. J'ai pu le voir à travers le CRP.»

Deux étudiants sont plus réticents sur la fonction de partage :

«Je trouve qu'on passe beaucoup de temps dessus et que cela n'apporte pas toujours quelque chose car les personnes qui répondent sont quasiment toujours du même avis que nous. Il est difficile d'ajouter de nouvelles idées. »

"Le carnet réflexif est intéressant et apporte du nouveau mais je n'aime pas spécialement que d'autres élèves, collègues lisent; je préfère discuter avec ceux avec qui j'ai des choses à partager et à débattre. Souvent l'avis des collègues était juste une approbation à notre idée (pensée). Rares étaient ceux qui la contredisaient. » 
44 L'importance du dialogue avec les pairs plus ou moins experts est conscientisée et appréciée même si les deux étudiants regrettent le manque de profondeur et de richesse des commentaires et de la controverse. Peut-être cela nécessite-t-il aussi un apprentissage car le dialogue écrit ne va pas de soi.

Le temps d'écriture est informel et varie selon les séances mais ne dépasse pas $15 \%$ de la durée de la séance. Trois étudiants auraient aimé consacrer plus de temps en cours à ces moments d'écriture qui permettent de "se remettre en question et mettre ses idées à plat ».

46 Si $90 \%$ des étudiants ont apprécié l'outil au terme du cours, $10 \%$ ne se le sont pas approprié car ils transfèrent leurs résistances et/ou leurs difficultés à écrire dans un cadre scolaire sur ce nouvel outil qui n'appelle pas d'évaluation sommative, tout au plus auto formative ou formatrice.

47 "J'ai trouvé très intéressant de se questionner sur ces différents thèmes. Mais j'étais frustrée car j'ai de la peine à m'exprimer par écrit. »

48 "Je n'ai pas aimé cette pratique. J'ai tendance à faire beaucoup de fautes d'orthographe, donc je n'aimais pas qu'on lise ce que j'écris. »

49 "J'ai moins aimé le travail dans le CR, car l'écriture spontanée n'est pas mon fort, il me faut du temps pour structurer ce que j'écris, sinon je réalise beaucoup de ratures. »

50 Perdure encore l'idée du produit fini, sans scorie, sans faute. L'écriture est plus envisagée comme un produit qu'un processus. En l'occurrence, l'étudiant qui sait qu'il sera lu focalise sur la forme comme il est courant de le faire dans l'évaluation de l'écriture scolaire. Il occulte aussi l'enjeu du dialogue d'idées et ne considère qu'une possible évaluation des pairs sur la forme de son écriture, notamment l'orthographe. On est loin d'une conception de l'écriture comme construction de sens.

51 Avant de proposer aux étudiants un retour réflexif sur le cours (voir le questionnaire en annexe 1), j'ai communiqué mon propre retour réflexif sur le cours que j'avais assuré en entier et écrit une sorte de lettre aux étudiants dont voici un extrait :

52 "A mon sens, l'enjeu était de vous faire réfléchir sur cette problématique cruciale de la réussite scolaire, de vous outiller au plan théorique et de faire en sorte que vous puissiez vous autoriser à penser en faisant des choix pertinents et conscientisés pour faire réussir les élèves qui vous seront confiés. Apprendre à penser et à faire penser ses élèves, c'est un enjeu fondamental pour un enseignant qui vit dans une société productiviste et consumériste où l'information et la communication priment parfois sur la connaissance. »

53 L'enjeu était de rendre les étudiants actrices et acteurs de leurs apprentissages et de leur formation. L'analyse met en évidence une plus-value pédagogique et didactique. Que les étudiants concernés en soient vivement remerciés, les parleurs, les causeurs, les taiseux, les résistants, les inquiets, les " cause toujours tu m'intéresses ", les scolaires, les actifs, les pédagogues, ${ }^{5}$ les passionnés, car toutes ces postures critiques m'ont permis d'évoluer dans ma pédagogie d'enseignante au tertiaire et de formatrice d'enseignants. C'était le moins pour un cours intitulé « Promouvoir un enseignement qui favorise la réussite scolaire ».

54 Un cours réussi repose sur la compréhension de ce que savent les étudiants (beaucoup plus que je ne crois souvent!), ce qui les interpelle, ce que je veux déconstruire (à la Derrida) et surtout construire dans l'interaction. Un cours raté, c'est un cours dont je 
ne sors pas enrichie, comme si la frontière n'avait pas été franchie et le voyage pas réalisé. C'est un cours où il n'y a pas eu de vraies questions, pas des questions dont je connais déjà la réponse. A mon sens, un cours réussi ouvre un espace de questions vives par les étudiants.

\section{Conclusion : un va-et-vient entre empathie et distanciation, une condition de la réflexivité}

Nul doute que le CRP soit un outil interactif qui permette à la fois l'empathie (se mettre à la place d'autrui pour comprendre son point de vue) mais aussi la distanciation (qui permet d'objectiver une expérience subjective) dans un espace heuristique toujours renouvelé. Cet espace est un lieu d'expérimentation des fonctions cognitive, réflexive et heuristique de l'écriture souvent réduite à une fonction scolaire, destinée à être évaluée ou à une visée strictement communicative. Le CRP alimente aussi le débat oral. Le CRP est un tremplin à des jeux de rôle où des argumentaires divers sont déployés à partir de questions vives et controversées concernant la pédagogie et l'éducation. Il s'agit d'apprendre l'empathie, condition nécessaire du débat démocratique et de la tolérance.

L'outil CRP concourt à la formation d'un praticien réflexif engagé et capable à la fois d'empathie et aussi de distanciation. In fine, il appert qu'une posture critique, auctoriale et réflexive conjointe favorise l'autonomisation des étudiants dans l'écriture en leur permettant d'associer vouloir et pouvoir.

\section{BIBLIOGRAPHY}

Bardin, L. (1998). L'analyse de contenu. Presses Universitaires de France.

Barré de Miniac, C. (2002). Le rapport à l'écriture. Une notion à plusieurs dimensions. Pratiques, 113-114, 29-40.

Blanchet, P. (2014). Nécessité d'une réflexion épistémologique. Dans P. Blanchet et p. Chardenet (dir.), Guide pour la recherche en didactique des langues et des cultures. Approches contextualisées ( $2^{\mathrm{e}}$ éd., chap.1, p. 5 à 15). Édition des archives contemporaines et Agence universitaire de la francophonie.

Dewey, J. (1968). Expérience et éducation. Armand Colin.

Goody, J. (1979). La raison graphique; La domestication de la pensée sauvage. Éditions de Minuit.

Lahire, B. (2004). La Culture des individus. Dissonances culturelles et distinction de soi. La Découverte.

Lebrun, M. (éd.) (2010). La classe de français et de littérature. EME intercommunications.

Lebrun, M. (2014). A propos du jugement de goût et de valeur : une question d'engagement. Dans J. Van Beveren (dir.), Littérature, langue et didactique. Mélanges offerts à Jean-Louis Dumortier, (p. 127-152). Presses universitaires de Namur. 
Langlade, G., et Rouxel, A. (dir.) (2004). Le Sujet lecteur. Lecture subjective et enseignement de la littérature. Presses Universitaires de Rennes.

Poisson, Y. (1983). L'approche qualitative et l'approche quantitative dans les recherches en éducation. Revue des sciences de l'éducation, 9(3), 369-378 https://www.erudit.org/fr/revues/rse/1983-v9n3-rse3487/900420ar.pdf

Schön, A-D. (1993). Le praticien réflexif. À la recherche du savoir caché dans l'agir professionnel. Éditions Logiques.

Wentzel, B. (2014). Internationalisation de la professionnalisation de l'enseignement : éléments d'analyse et de synthèse. Dans B. Wentzel, V. Lussi et R. Borer (dir.), Professionnalisation de la formation des enseignants : perspectives internationales (p. 109-127). Presses universitaires de Lorraine.

\section{NOTES}

1. Pour des raisons d'éthique les prénoms des étudiants ont été changés. Ceux-ci ont d'ailleurs renseigné le questionnaire (annexe 1) anonymement.

2. Il s'agit d'un cours de niveau licence donnant lieu à 3 crédits et dont j'assume l'entièreté des séances liées.

3. Cet outil de traitement statistique permet de traiter des réponses à des questions ouvertes : SPSS Text Analysis for Surveys : IBM SPSS Text Analytics for Surveys 4.0

4. Les verbatims ont été sélectionnés dans le retour réflexif sur l'outil réalisé par une cohorte de 28 étudiants suisses en formation au Bachelor de l'enseignement primaire à la HEP-BEJUNE pendant l'année académique 2016-2017.

5. Certains étudiants ont mis en œuvre l'outil dans leurs classes de stage tout en l'adaptant à leur jeune public et d'autres ont envie aussi de l'essayer.

\section{ABSTRACTS}

This contribution analyzes the interest of a reflexivity tool for tertiary education and teacher training by presenting its ins and outs while giving the floor to the users, students in training for the primary or secondary education, because it is them, in fine, who are the best able to give thought on what is at stake in the writing of a shared reflexive notebook (now CRP). The analysis of their critical comments reveals the adequacy or the distortion between what is desired and what is experienced by the students through a heuristic and shared mode of writing. The tool seems to foster both a stance of empathy and distance that gives meaning to reflexivity and writing.

La contribution analyse l'intérêt d'un outil de réflexivité pour l'enseignement tertiaire et la formation à l'enseignement : il s'agit de présenter ses tenants et aboutissants tout en donnant la parole à ses utilisateurs, des étudiants en formation à l'enseignement primaire ou secondaire, car ce sont elles et eux, in fine, qui sont le plus à même de donner à penser sur ce qui est en jeu dans l'écriture d'un carnet réflexif partagé (désormais CRP). L'analyse de leurs commentaires critiques 
permet d'appréhender l'adéquation ou la distorsion entre ce qui est souhaité et ce qui est vécu par ce corps estudiantin à travers un mode d'écriture heuristique et partagée. L'outil favorise à la fois une posture d'empathie et une posture de distanciation qui donne du sens à la réflexivité et à l'écriture.

INDEX

Mots-clés: réflexivité, partage, critique, outil d'écriture, heuristique

\section{AUTHOR}

\section{MARLĖNE LEBRUN}

HEP-BeJuNe, Suisse, Marlene.Lebrun@hep-bejune.ch 